\title{
Coronary Artery Calcification in Hemodialysis and Peritoneal Dialysis
}

\author{
Thijs.T Jansz $^{a} \quad$ Franka E. van Reekum ${ }^{a} \quad$ Akin Özyilmaz $^{\text {b, c }}$ Pim A. de Jong ${ }^{d}$ \\ Franciscus T.J. Boereboom $^{\mathrm{e}} \mathrm{f}^{\mathrm{f}}$ Tiny Hoekstra ${ }^{g}$ Marianne C. Verhaar ${ }^{\mathrm{a}}$ \\ Brigit C. van Jaarsveld ${ }^{\mathrm{e}} \mathrm{g}$
}

${ }^{a}$ Department of Nephrology and Hypertension, University Medical Center Utrecht, Utrecht University, Utrecht, The Netherlands; ${ }^{b}$ Division of Nephrology, Department of Internal Medicine, University Medical Center Groningen, Groningen, The Netherlands; ' Dialysis Center Groningen, Groningen, The Netherlands; ${ }^{\mathrm{C}}$ Department of Radiology, University Medical Center Utrecht, Utrecht, The Netherlands; ${ }^{e}$ Dianet Dialysis Centers, Utrecht, The Netherlands; fDepartment of Internal Medicine, Diakonessenhuis Utrecht, Utrecht, The Netherlands; ${ }^{9}$ Department of Nephrology, Amsterdam UMC, Vrije Universiteit Amsterdam, Amsterdam, The Netherlands

\section{Keywords}

Coronary artery calcification · Hemodialysis · Peritoneal dialysis

\begin{abstract}
Background: Vascular calcification is seen in most patients on dialysis and is strongly associated with cardiovascular mortality. Vascular calcification is promoted by phosphate, which generally reaches higher levels in hemodialysis than in peritoneal dialysis. However, whether vascular calcification develops less in peritoneal dialysis than in hemodialysis is currently unknown. Therefore, we compared coronary artery calcification (CAC), its progression, and calcification biomarkers between patients on hemodialysis and peritoneal dialysis. Methods: We measured CAC in 134 patients who had been treated exclusively with hemodialysis $(n=94)$ or peritoneal dialysis $(n=40)$ and were transplantation candidates. In 57 of them (34 on hemodialysis and 23 on peritoneal dialysis), we also measured CAC progression annually up to 3 years and the inactive species of desphospho-uncarboxylated matrix Gla protein (dp-ucMGP), fetuin-A, osteo-
\end{abstract}

protegerin. We compared CAC cross-sectionally with Tobit regression. CAC progression was compared in 2 ways: with linear mixed models as the difference in square root transformed volume score per year ( $\triangle$ CAC SQRV) and with Tobit mixed models. We adjusted for potential confounders. $R \boldsymbol{e}$ sults: In the cross-sectional cohort, CAC volume scores were $92 \mathrm{~mm}^{3}$ in hemodialysis and $492 \mathrm{~mm}^{3}$ in peritoneal dialysis (adjusted difference $436 \mathrm{~mm}^{3} ; 95 \% \mathrm{Cl}-47$ to $919 ; p=0.08$ ). In the longitudinal cohort, peritoneal dialysis was associated with significantly more CAC progression defined as $\triangle C A C$ SQRV (adjusted difference $1.20 ; 95 \% \mathrm{Cl} 0.09$ to $2.31 ; p=0.03$ ), but not with Tobit mixed models (adjusted difference in CAC score increase per year $106 \mathrm{~mm}^{3} ; 95 \% \mathrm{Cl}-140$ to $352 ; p=$ $0.40)$. Peritoneal dialysis was associated with higher osteoprotegerin (adjusted $p=0.02$ ) but not with dp-ucMGP or fetuin-A. Conclusions: Peritoneal dialysis is not associated with less CAC or CAC progression than hemodialysis, and perhaps with even more progression. This indicates that vascular calcification does not develop less in peritoneal dialysis than in hemodialysis.

C 2018 The Author(s) Published by S. Karger AG, Basel

\begin{tabular}{ll}
\hline KARGER & $\begin{array}{l}\text { () } 2018 \text { The Author(s) } \\
\text { Published by S. Karger AG, Basel }\end{array}$ \\
E-Marger \\
E-Mail karger@karger.com & This article is licensed under the Creative Commons Attribution- \\
www.karger.com/ajn & $\begin{array}{l}\text { NonCommercial-NoDerivatives 4.0 International License (CC BY- } \\
\text { NC-ND) (http://www.karger.com/Services/OpenAccessLicense). } \\
\text { Usage and distribution for commercial purposes as well as any dis- } \\
\text { tribution of modified material requires written permission. }\end{array}$
\end{tabular}




\section{Introduction}

Cardiovascular disease is the leading cause of death among patients with end-stage renal disease $[1,2]$. This high cardiovascular mortality is strongly associated with vascular calcification [3,4], which occurs frequently [2] and progresses almost universally in end-stage renal disease [5]. Vascular calcification can be measured at various sites, such as the coronary arteries, and is promoted by phosphate, which is frequently elevated in end-stage renal disease $[6,7]$.

Remarkably, it is unknown whether vascular calcification is affected by dialysis modality, of which the 2 major types are hemodialysis and peritoneal dialysis. In theory, peritoneal dialysis might induce less vascular calcification than hemodialysis because patients on peritoneal dialysis generally have lower serum phosphate [8] probably owing to their continuous clearance. However, there have never been randomized studies on this subject, as randomization to dialysis modality is generally refused by patients [9]. Moreover, patients on peritoneal dialysis are typically younger and healthier due to the prerequisites of treatment at home [10], which has hampered previous observational research that did not attempt to statistically adjust for this [11-14].

To overcome this, we compared patients treated with hemodialysis or peritoneal dialysis who were all eligible for transplantation and thus relatively comparable in age and comorbidities. First, we compared coronary artery calcification (CAC) cross-sectionally between prevalent patients who had been treated exclusively with hemodialysis or peritoneal dialysis. Second, we compared CAC progression up to 3 years among those who underwent follow-up measurements. Additionally, we studied calcification biomarkers in relation to CAC progression, and compared these between patients on hemodialysis and peritoneal dialysis.

\section{Methods}

\section{Cross-Sectional Cohort}

We analyzed a cross-sectional sample of patients that had been treated exclusively with conventional hemodialysis or peritoneal dialysis and participated in the NOCTx study. NOCTx (NCT00950573) is a prospective nonrandomized study that included patients on chronic conventional hemodialysis or peritoneal dialysis with a minimum dialysis vintage of 2 months, patients who switched to nocturnal hemodialysis, and patients who received a kidney transplant. Thus, all patients had been treated with hemodialysis or peritoneal dialysis at inclusion. Patients were eligible when aged between 18 and 75 years and were candidates for transplantation when on dialysis. NOCTx excluded patients with a life expectancy $<3$ months, non-adherence to dialysis regimens, drug abuse, and pregnancy.

Between December 2009 and February 2016, 329 patients were screened for eligibility in 8 Dutch dialysis centers. NOCTx included 181 of these patients, of whom 135 were being treated with hemodialysis and 46 with peritoneal dialysis at inclusion. We excluded patients who were treated with hemodialysis $>16 \mathrm{~h}$ per week $(n=14)$, as we theorized that more intensive dialysis regimens might mitigate calcification. Furthermore, we excluded patients who had a history of treatment with the other modality of over 3 months $(n=33)$, leaving a sample of 134 patients.

\section{Longitudinal Cohort}

We analyzed a longitudinal sample of patients from the NOCTx study who continued treatment with conventional hemodialysis or peritoneal dialysis after inclusion and completed at least one follow-up visit $(n=57)$. In NOCTx, CAC was measured at inclusion, and after 1,2 and 3 years. Also, blood was collected in $4.5 \mathrm{~mL}$ potassium-ethylenediaminetetraacetic acid vacutainers (on a nondialysis day in case of hemodialysis), immediately centrifuged and stored in aliquots at $-80^{\circ} \mathrm{C}$ without thawing at inclusion. Patients left the study if their renal replacement therapy was changed.

\section{Treatment Characteristics}

Patients were treated according to the Kidney Disease: Improving Global Outcomes guidelines by the attending nephrologists [15]. Hemodialysis was delivered 3 times a week for $4 \mathrm{~h}$ with a default $1.50 \mathrm{mmol} / \mathrm{L}$ dialysate calcium concentration, and peritoneal dialysis as automatic or continuous ambulant peritoneal dialysis with a default $1.25 \mathrm{mmol} / \mathrm{L}$ dialysate calcium concentration.

\section{CAC Measurements}

We determined CAC scores on nonenhanced, prospectively triggered cardiac multi-slice computed tomography (iCT 256, Philips Healthcare, Best, The Netherlands). Acquisition parameters were as follows: $120 \mathrm{kV}, 40-50 \mathrm{mAs}$, rotation time $270 \mathrm{~ms}$, and $128 \times 0.625 \mathrm{~mm}$ collimation. Metoprolol was administered intravenously if heart rate was above $60 / \mathrm{min}$ to improve imaging quality. We used a calcium threshold of $\geq 130$ Hounsfield units. A single observer (T.T.J.) read all scans chronologically per patient in order to exclude segments with severe motion artefacts or stents at a given scan from an entire set. Using commercially available software (Heartbeat CS, Philips Healthcare, The Netherlands), we calculated calcium volume scores. Reproducibility of CAC measurements has been shown to be excellent, with an intraclass correlation coefficient of $>0.95[16]$.

\section{Calcification Biomarker Measurements}

Plasma levels of desphospho-uncarboxylated matrix Gla protein (dp-ucMGP) were determined as described before [17]. The within-run and total variations of this assay were $0.8-6.2 \%$ and $3.0-8.2 \%$, respectively. The assay measuring range was between 300 and $12,000 \mathrm{pmol} / \mathrm{L}$ and was linear up to $11,651 \mathrm{pmol} / \mathrm{L}$ [18]. The dp-ucMGP assays were performed in a single run by the laboratory of Coagulation Profile, department of Biochemistry, Maastricht, The Netherlands. Plasma fetuin-A and osteoprotegerin levels were measured with a Bio-Plex system (Bio-Rad) multiplex assay by the laboratory of the University Medical Center Utrecht, Utrecht, The Netherlands. All assays were executed in a single run. 
Other Study Variables

Study personnel recorded demographic and clinical parameters at inclusion (pre-dialysis blood pressure and post-dialysis weight averaged from routine measurements during 3 hemodialysis sessions or 2 outpatient visits in case of peritoneal dialysis). Laboratory parameters (total calcium, albumin, phosphate, C-reactive protein, and parathyroid hormone) were obtained at inclusion by averaging routine measurements from 3 months, performed with standard laboratory techniques at the local treatment facilities. We classified residual urine production as present $(\geq 100$ $\mathrm{mL} / 24 \mathrm{~h}$ ) or absent. We defined dialysis vintage as the time between the first day of dialysis and the day of scanning, minus the time with a functioning kidney transplant.

\section{Statistical Analyses}

We reported normally distributed variables as mean \pm SD, nonnormally distributed variables as median (interquartile range $[\mathrm{IQR}]$ ), and categorical data as number (percentage). We compared normally distributed variables with Student $t$ tests, non-normally distributed variables with Mann-Whitney-U tests, and categorical data with chi-square tests.

We compared CAC volume scores cross-sectionally with Tobit regression. Tobit regression can be used to analyze variables with floor and/or ceiling effects [19]. This may be the case with CAC scores, when calcification can be present below the detection limit while the CAC score is 0 . With Tobit regression, we assume our outcome variable is actually a normally distributed variable that has been truncated (here CAC score truncated at zero). By modeling this latent underlying variable, values of zero do not need to be excluded from the analyses and do not severely skew the results $[19,20]$.

To compare CAC progression, we used 2 different approaches, since a valid standard method to analyze CAC progression is lacking. First, we analyzed CAC progression with linear mixed models as change per year in square root transformed volume scores ( $\triangle$ CAC SQRV). This approach, also known as Hokanson's method, accounts for interscan variability [21] and has been used by others [22]. We adjusted these analyses for CAC SQRV at inclusion. Second, we used Tobit mixed models to analyze CAC progression. We adjusted for factors known to induce calcification [23]: age (years), sex (male/female), presence of diabetes mellitus (yes/no), dialysis vintage (months), presence of residual urine production $\geq 100$ $\mathrm{mL} / 24 \mathrm{~h}$ (yes/no), and vitamin $\mathrm{K}$ antagonist use (yes/no).

We used linear regression to compare biomarker levels between dialysis modalities. Dp-ucMGP levels were log-transformed, as these were right-skewed. We adjusted for potential confounders as described above. To determine the relationship between biomarkers and $\triangle \mathrm{CAC}$ SQRV between inclusion and 1 year, we calculated Pearson's correlation coefficients.

We report regression coefficients with $95 \%$ CI. We considered $p$ values $\leq 0.05$ (2-tailed) statistically significant and used R 3.4.1 ( $R$ Foundation Statistical Computing) for all analyses.

\section{Results}

\section{Cross-Sectional Cohort}

The cross-sectional cohort included 134 patients who had been treated exclusively with hemodialysis $(n=94)$ or peritoneal dialysis $(n=40)$. The mean age of this cohort was $54 \pm 12$ years, $94(70 \%)$ were male, median dialysis vintage was 17 (IQR 10-34) months, and 24 (18\%) had diabetes mellitus. The patients on hemodialysis had a median 6-month longer dialysis vintage, were somewhat heavier, had higher blood pressures, and had lower calcium and higher albumin levels than the patients on peritoneal dialysis (Table 1). Phosphate levels were not significantly higher in the patients on hemodialysis.

CAC volume scores were 92 (IQR 1-663) in the patients on hemodialysis and 492 (IQR 92-1,139) in the patients on peritoneal dialysis. The distribution of the CAC volume scores is illustrated by a smoothed version of a histogram (Kernel density plot) in online supplementary Figure S1 (for all online suppl. material, see www.karger. com/doi/10.1159/000494665). In Tobit regression, the CAC volume scores were not significantly $(p=0.15)$ higher in patients on peritoneal dialysis compared to patients on hemodialysis (difference $342 \mathrm{~mm}^{3}$; $95 \% \mathrm{CI}-125$ to 808 ). When adjusted for age, sex, diabetes mellitus, dialysis vintage, residual urine production, and vitamin $\mathrm{K}$ antagonist use, peritoneal dialysis was also not significantly $(p=0.08)$ associated with more CAC than hemodialysis (difference $436 \mathrm{~mm}^{3}$; 95\% CI -47 to 919 ).

\section{Longitudinal Cohort}

The longitudinal cohort included 57 patients treated with hemodialysis $(n=34)$ or peritoneal dialysis $(n=23)$ who completed at least one follow-up visit. The mean age of this cohort was $52 \pm 13$ years, 37 (65\%) were male, median dialysis vintage was 17 (IQR 8-47) months, and 7 $(12 \%)$ had diabetes mellitus. The patients on hemodialysis were somewhat heavier, had higher systolic blood pressures, tended to have longer dialysis vintages, tended to use more vitamin K antagonists, and had lower calcium and higher albumin levels than the patients on peritoneal dialysis (Table 1), whereas their other characteristics were comparable. Notably, CAC volume scores at inclusion were not significantly different between patients on hemodialysis (median 163, IQR 5-745) and patients on peritoneal dialysis (median 76, IQR 2-696, $p=0.68$ ), nor was the proportion of patients with zero calcification $(n=$ $8,24 \%$ vs. $n=6,26 \%$, respectively, $p=0.99$ ). There were also no significant differences between the longitudinal cohort $(n=57)$ and those who underwent treatment with hemodialysis or peritoneal dialysis after inclusion but did not undergo follow-up CAC measurements (online suppl. Table S1).

The maximum follow-up duration was 1 year for $25 \mathrm{pa}-$ tients, 2 years for 18 patients, and 3 years for 14 patients. 
Table 1. Characteristics of the 134 patients included in the cross-sectional CAC analysis and of the 57 patients included in the CAC progression analyses, stratified by dialysis modality

\begin{tabular}{|c|c|c|c|c|c|}
\hline \multicolumn{2}{|c|}{ Cross-sectional cohort } & \multirow{2}{*}{$\begin{array}{l}p \text { for } \\
\text { difference }\end{array}$} & \multicolumn{2}{|c|}{ Longitudinal cohort } & \multirow{2}{*}{$\begin{array}{l}p \text { for } \\
\text { difference }\end{array}$} \\
\hline $\begin{array}{l}\text { hemodialysis } \\
(n=94)\end{array}$ & $\begin{array}{l}\text { peritoneal } \\
\text { dialysis } \\
(n=40)\end{array}$ & & $\begin{array}{l}\text { hemodialysis } \\
(n=34)\end{array}$ & $\begin{array}{l}\text { peritoneal } \\
\text { dialysis } \\
(n=23)\end{array}$ & \\
\hline
\end{tabular}

Demographics and medical history

Age, years

Male, $n$ (\%)

Body mass index, $\mathrm{kg} / \mathrm{m}^{2}$

Systolic blood pressure, $\mathrm{mm} \mathrm{Hg}$

Diastolic blood pressure, $\mathrm{mm} \mathrm{Hg}$

Diabetes mellitus, $n$ (\%)

Cardiovascular disease, $n(\%)$

Current smoker, $n$ (\%)

$\begin{array}{cc}56 \pm 11 & 51 \pm 15 \\ 62(66) & 32(80) \\ 26.2 \pm 4.4 & 24.7 \pm 3.3 \\ 143 \pm 20 & 135 \pm 13 \\ 79 \pm 12 & 85 \pm 10 \\ 21(22) & 3(8) \\ 23(25) & 10(25) \\ 13(14) & 5(13)\end{array}$

$\begin{array}{rc}0.06 & 54 \pm 12 \\ 0.16 & 20(59) \\ 0.05 & 26.7 \pm 4.9 \\ 0.03 & 142 \pm 20 \\ <0.01 & 79 \pm 11 \\ 0.07 & 6(18) \\ 0.99 & 7(21) \\ 0.99 & 4(12)\end{array}$

$\begin{array}{cc}49 \pm 14 & 0.17 \\ 17(74) & 0.37 \\ 24.5 \pm 3.4 & 0.04 \\ 132 \pm 18 & 0.05 \\ 82 \pm 14 & 0.29 \\ 1(4) & 0.28 \\ 5(22) & 0.99 \\ 1(4) & 0.62\end{array}$

History of kidney disease

Dialysis vintage, months

Cause of end-stage renal disease, $n(\%)$

Cystic kidney disease

Interstitial nephritis

Glomerulonephritis

Vascular disease

Diabetic nephropathy

Other

Unknown

$\begin{array}{cc}19(11-35) & 13(7-31) \\ 18(19) & 6(15) \\ 5(5) & 1(3) \\ 24(26) & 7(18) \\ 21(22) & 11(28) \\ 10(11) & 2(5) \\ 8(9) & 7(18) \\ 8(9) & 6(15)\end{array}$

$\begin{array}{lll}0.05 & 26(10-56) & 12(5-47) \\ 0.42 & & \\ & 4(12) & 5(22) \\ & 2(6) & 1(4) \\ 9(26) & 5(22) \\ & 8(24) & 5(22) \\ & 5(15) & 1(4) \\ 4(12) & 6(26) \\ & 2(6) & 0\end{array}$

Dialysis therapy and kidney function

Dialysis therapy

Weekly hemodialysis hours

Daily peritoneal dialysis dwells

Daily peritoneal dialysis volume, $\mathrm{L}$

$3.1+0.5$

$\begin{array}{ll}- & 4.4 \pm 0.6\end{array}$

Kidney function

Residual urine production $\geq 100 \mathrm{~mL} / 24 \mathrm{~h} n$ (\%)

Medication use*

Vitamin K antagonist, $n(\%)$

Vitamin D analogue, $n(\%)$

Calcium-containing phosphate binder, $n(\%)$

Cinacalcet, $n(\%)$

Laboratory parameters

Calcium, $\mathrm{mmol} / \mathrm{L}$

Albumin, g/L

Phosphate, $\mathrm{mmol} / \mathrm{L}$

C-reactive protein, $\mathrm{mg} / \mathrm{L}$

Parathyroid hormone, pmol/L

$\begin{array}{ll}- & 4.4 \pm 0.6 \\ - & 9.8 \pm 2.4\end{array}$

-
-
$4.4 \pm 0.6$
$9.8 \pm 2.4$

$55(59) \quad 29(73)$
$2.9 \pm 0.3$

$11.0 \pm 2.0$

$\begin{array}{ll}- & 4.3 \pm 0.6 \\ - & 9.0 \pm 2.2\end{array}$

$0.18 \quad 20(59) \quad 14(61)$

0.99

$\begin{array}{lrrrrr}12(15) & 1(3) & 0.11 & 7(21) & 0 & 0.06 \\ 61(75) & 32(89) & 0.15 & 29(85) & 19(83) & 0.99 \\ 28(35) & 14(39) & 0.81 & 18(53) & 10(44) & 0.67 \\ 16(20) & 7(19) & 0.99 & 8(24) & 3(13) & 0.52\end{array}$

$\begin{array}{cccc}2.3 \pm 0.1 & 2.4 \pm 0.1 & 0.01 & 2.3 \pm 0.1 \\ 41.4 \pm 3.2 & 38.3 \pm 3.6 & <0.001 & 40.8 \pm 3.0 \\ 1.6 \pm 0.4 & 1.6 \pm 0.4 & 0.69 & 1.6 \pm 0.3 \\ 3(2-6) & 3(1-13) & 0.93 & 2(2-7) \\ 22(15-41) & 22(14-37) & 0.53 & 30(17-48)\end{array}$

$\begin{array}{cc}2.4 \pm 0.1 & 0.01 \\ 38.5 \pm 3.5 & 0.02 \\ 1.6 \pm 0.3 & 0.50 \\ 2(1-18) & 0.91 \\ 22(15-41) & 0.53\end{array}$

Data are presented as mean \pm SD, median (IQR) or number (\%).

* In the cross-sectional cohort, data on medication use were available in 81 patients on hemodialysis and 36 patients on peritoneal dialysis; in the longitudinal cohort data on medication use were available in all patients.

CAC, coronary artery calcification.

CAC progressed in most patients, but only in 2 of 8 patients on hemodialysis without CAC at inclusion, and in 2 of 6 patients on peritoneal dialysis without $\mathrm{CAC}$ at inclusion (Fig. 1). We analyzed CAC progression as $\triangle \mathrm{CAC}$ SQRV with linear mixed models and with Tobit mixed models.
CAC progressed with $1.72 \triangle \mathrm{CAC}$ SQRV per year in patients on hemodialysis (95\% CI 0.81 to 2.64 ) and with $2.73 \triangle \mathrm{CAC} S Q R V$ per year in patients on peritoneal dialysis (95\% CI 1.58 to 3.88; Fig. 2). As can be seen in Table 2 , peritoneal dialysis was not significantly $(p=0.18)$ as- 
Fig. 1. CAC progression in 57 patients on dialysis stratified by dialysis modality, depicted as individual trajectories of calcium volume scores. Individual trajectories of change in calcium volume score in patients on hemodialysis (left panel) and patients on peritoneal dialysis (right panel). Trajectories of 2 patients on hemodialysis and one on peritoneal dialysis with scores $>$ 5,000 are not shown in this figure. Number of patients shown at $0,1,2$, and 3 years: hemodialysis $32,32,21$, and 10 ; peritoneal dialysis: 22, 22, 7, and 4. Note that lines may overlap around 0 .

Fig. 2. CAC progression per year in 57 patients on dialysis stratified by dialysis modality, depicted as boxplots of change in square root transformed volume score. Change in square root transformed volume scores (Y-axis) stratified by dialysis modality (X-axis) as boxplots. Note that square root transformations cannot be backtransformed. Number of patients per group per period $(\mathrm{N})$ denoted below the boxplots. Crude $\mathrm{P}$ for difference in change in square root transformed volume score per year: 0.18 ; adjusted $\mathrm{P}$ for difference in change in square root transformed volume score per year: 0.03 .
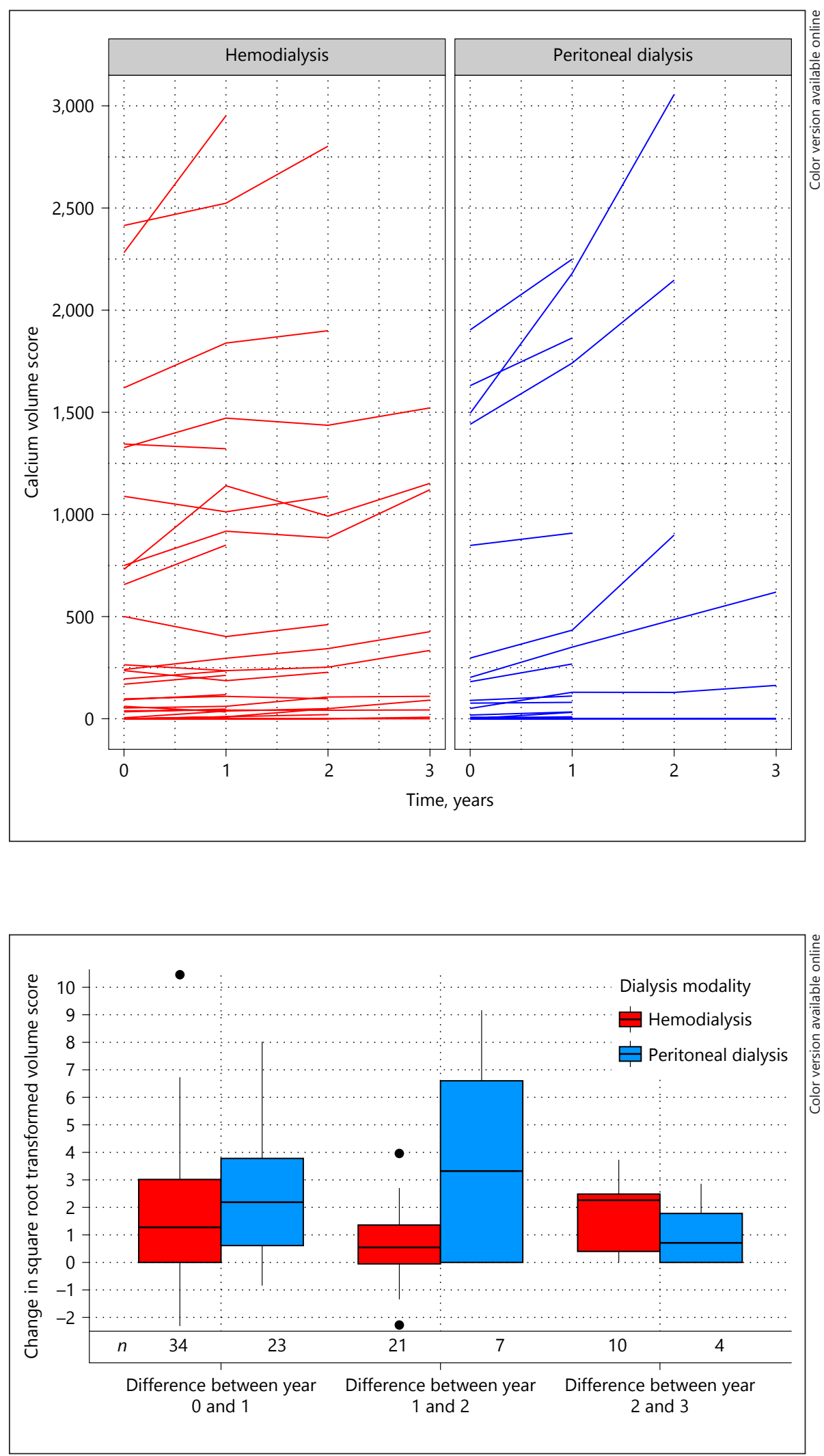
Table 2. Effect estimates of CAC progression for peritoneal dialysis $(n=23)$ compared to hemodialysis $(n=34)$ analyzed with linear mixed models as $\triangle \mathrm{CAC}$ SQRV and with Tobit mixed models, with different multivariate adjustments

\begin{tabular}{lllll}
\hline & Unadjusted & $\begin{array}{l}\text { CAC SQRV } \\
\text { at inclusion* }\end{array}$ & $\begin{array}{l}\text { Adjustment for age } \\
\text { and gender }\end{array}$ & Full adjustment \\
\hline $\begin{array}{l}\text { aCAC SQRV } \\
\text { Tobit regression }\end{array}$ & $\begin{array}{c}1.01(-0.47 \text { to } 2.47) \\
189(-81 \text { to } 459)\end{array}$ & $\begin{array}{c}1.22(0.16 \text { to } 2.29) \\
-\end{array}$ & $\begin{array}{r}1.25(0.19 \text { to } 2.33) \\
77(-184 \text { to } 338)\end{array}$ & $\begin{array}{r}1.20(0.09 \text { to } 2.31) \\
106(-140 \text { to } 352)\end{array}$ \\
\hline
\end{tabular}

*Tobit mixed models could not be adjusted for CAC SQRV at inclusion.

${ }^{\dagger}$ The linear mixed models of $\triangle \mathrm{CAC}$ SQRV were additionally adjusted for CAC SQRV at inclusion.

${ }^{\ddagger}$ Full adjustment included age, sex, diabetes mellitus, dialysis vintage, presence of residual urine production, and vitamin

$\mathrm{K}$ antagonist use. The linear mixed models of $\triangle \mathrm{CAC}$ SQRV were additionally adjusted for CAC SQRV at inclusion

95\% CI between brackets.

CAC, coronary artery calcification.

sociated with higher $\triangle \mathrm{CAC}$ SQRV than hemodialysis in unadjusted analyses (difference in $\triangle \mathrm{CAC}$ SQRV per year $1.01 ; 95 \%$ CI -0.47 to 2.47 ). When adjusted for CAC SQRV at inclusion, age, sex, diabetes mellitus, dialysis vintage, vitamin $\mathrm{K}$ antagonist use, and presence of residual urine production, peritoneal dialysis was significantly $(p=0.03)$ associated with $1.20 \Delta$ CAC SQRV per year higher CAC progression compared to hemodialysis ( $95 \%$ CI 0.09-2.31).

In Tobit mixed models, CAC progressed with $99 \mathrm{~mm}^{3}$ per year in hemodialysis (95\% CI -42 to 240 ) and with $288 \mathrm{~mm}^{3}$ per year in peritoneal dialysis (95\% CI 57 to 519). As can be seen in Table 2, peritoneal dialysis was not significantly associated with higher CAC progression than hemodialysis in both crude and adjusted analyses with Tobit mixed models (crude difference $189 \mathrm{~mm}^{3}$ per year; $95 \% \mathrm{CI}-81$ to $459 ; p=0.17$; and fully adjusted difference $106 \mathrm{~mm}^{3}$ per year; $95 \% \mathrm{CI}-140$ to $352 ; p=0.40$ ).

\section{Calcification Biomarkers}

At inclusion, we measured calcification biomarkers in the longitudinal cohort $(n=57)$. Dp-ucMGP levels were median 1,689 (IQR 1,304-3,470) pmol/L in hemodialysis, and 1,548 (IQR 900-1,822) $\mathrm{pmol} / \mathrm{L}$ in peritoneal dialysis. Fetuin-A levels were mean $0.20 \pm 0.06 \mathrm{~g} / \mathrm{L}$ in hemodialysis and $0.21 \pm 0.08 \mathrm{~g} / \mathrm{L}$ in peritoneal dialysis. Osteoprotegerin were mean $3.2 \pm 1.4 \mu \mathrm{g} / \mathrm{L}$ in hemodialysis and $3.3 \pm$ $1.2 \mu \mathrm{g} / \mathrm{L}$ in peritoneal dialysis. In univariate analyses, peritoneal dialysis was not associated with differences in dp-ucMGP, fetuin-A, or osteoprotegerin levels (online suppl. Table S2). When adjusted for age, sex, diabetes mellitus, dialysis vintage, vitamin $\mathrm{K}$ antagonist use, and presence of residual urine production, peritoneal dialysis was associated with $0.83 \mu \mathrm{g} / \mathrm{L}$ higher osteoprotegerin lev- els than hemodialysis (95\% CI 0.13 to $1.52 ; p=0.02$ ), but not with differences in dp-ucMGP or fetuin-A. Osteoprotegerin correlated with $\triangle$ CAC SQRV (Pearson's correlation coefficient $0.32, p=0.05$ ), while dp-ucMGP and fetuin-A did not correlate with $\triangle \mathrm{CAC}$ SQRV (Pearson's correlation coefficients $0.23, p=0.13$; and $-0.01, p=0.93$ ).

\section{Discussion}

Our study investigated whether vascular calcification develops less in peritoneal dialysis than in hemodialysis, cross-sectionally and longitudinally. In a large cross-sectional cohort, we found that patients treated with peritoneal dialysis do not have less CAC than patients treated with hemodialysis. In the longitudinal cohort, we found that patients on peritoneal dialysis do not have less CAC progression than patients on hemodialysis. Altogether, this indicates that vascular calcification does not develop less in peritoneal dialysis than in hemodialysis.

Few studies have compared vascular calcification between hemodialysis and peritoneal dialysis, that is, 3 cross-sectional studies and one longitudinal study. An American cross-sectional study found more frequent CAC in pediatric patients on hemodialysis ( $9 / 21$ patients) than on peritoneal dialysis (2/17 patients) [11]. An Albanian cross-sectional study found more frequent cardiac valve calcification in adult patients on hemodialysis (24/34 patients) than on peritoneal dialysis (10/30 patients) [12]. A Korean cross-sectional study did not find a significant difference in CAC score between patients on hemodialysis $(n=31$, median score 30$)$ and those on peritoneal dialysis ( $n=15$, median score 16) [13]. Finally, a Taiwanese study did not find significant differences in 
CAC score or one-year CAC progression between patients on hemodialysis $(n=18$, median score increased from 110 to 175$)$ and patients on peritoneal dialysis ( $n=$ 15 , median score increased from 3 to 76) [14, 24]. However, all of these studies had imbalances in age, dialysis vintage, or comorbidities between groups, which were not adjusted for statistically. In addition, the longitudinal study analyzed CAC progression as percentage change, which yields biased results because of the skewed distribution of CAC scores with excessive zeros [5].

The problematic distribution of CAC scores precludes many strategies commonly used to analyze changes. This is also why a standard method to analyze CAC progression is lacking. We therefore chose to analyze CAC progression with 2 approaches that are valid from a statistical viewpoint: as $\triangle \mathrm{CAC}$ SQRV with linear mixed models and with Tobit mixed models. Our data showed a significant effect with the former approach that appears larger than that with the latter approach. In fact, if we maintained the definition of CAC progression $\geq 2.5 \triangle$ CAC SQRV by Hokanson et al. [21], there would be 6 out of 34 patients on hemodialysis with progression and 9 out of 23 patients on peritoneal dialysis with progression, implying a number needed to harm of 4.7 patients to make one more patient progress in CAC on peritoneal dialysis than on hemodialysis. However, this larger effect may be due to modest right-skewness of $\triangle \mathrm{CAC} S \mathrm{SQV}$, despite the square root transformation. On the other hand, this larger effect may be because Tobit mixed models could not be adjusted for CAC score at inclusion. Nevertheless, the estimates from both analyses are in the same direction, which support our conclusion that peritoneal dialysis is not associated with less CAC progression than hemodialysis.

There could be some explanations for our unexpected finding that peritoneal dialysis is not associated with less CAC or CAC progression. On the one hand, patients on peritoneal dialysis may have had a greater time-averaged exposure to phosphate than patients on hemodialysis. After all, time-averaged phosphate exposure in hemodialysis is lower than suggested by pre-dialysis phosphate levels because of the sawtooth pattern in hemodialysis [25]. On the other hand, there could have been differences in calcium balance. Patients on hemodialysis might have had a positive calcium balance with the default dialysate calcium concentration of $1.50 \mathrm{mmol} / \mathrm{L}$ based on kinetic modeling studies [26], but it is unknown whether patients on peritoneal dialysis also had a positive calcium balance with a default dialysate calcium concentration of 1.25 $\mathrm{mmol} / \mathrm{L}$, as this also depends on ultrafiltration [27]. This would require detailed calcium balance studies.
Our findings regarding the 3 calcification biomarkers do not allow firm conclusions and need further exploration. First, we found that osteoprotegerin was associated with progression of CAC, in accordance with previous studies $[28,29]$. Nevertheless, osteoprotegerin should theoretically protect against vascular calcification by preventing the receptor activator of nuclear factor- $\kappa \mathrm{B}$ ligand from binding to the RANK receptor [30]. Whether our finding indicates a compensatory response requires additional study. Second, we did not find a significant association between CAC progression and dp-ucMGP. Dp-ucMGP is an inverse marker of calcification inhibition potential, as its active form inhibits vascular calcification after carboxylation by vitamin $\mathrm{K}$ [31]. It is possible that larger samples are needed to study the relationship between CAC progression and dp-ucMGP. Third, we did not find any relationship between fetuin-A and CAC progression. Fetuin-A is a hepatic protein that forms soluble complexes with calcium and phosphate (calciprotein particles [CPPs]) and thus prevents calcification [32]. The reason we did not find a relationship with CAC progression lies probably in these CPPs: an ordinary fetuin-A measurement includes the CPP-bound fetuin-A, which is the fetuin-A fraction that has already been used up. Future studies should measure the non-CPP-bound fraction of fetuin-A after an extra centrifugation step [33], or should measure CPPs directly [34].

Our results should be interpreted within certain limitations. The size of our longitudinal cohort was small and patients on peritoneal dialysis had a limited follow-up duration. Larger studies are necessary to investigate whether peritoneal dialysis may be associated with more CAC than hemodialysis and to investigate the relationship between calcification biomarkers and CAC progression. Also, our study was non-randomized, although randomization to dialysis modalities has proven infeasible in earlier studies [9].

Our study has several strengths as well. This study is the largest so far to compare vascular calcification between hemodialysis and peritoneal dialysis, combining a large cross-sectional cohort with follow-up data on progression of CAC. Also, we accounted for the skewness and zero-inflation of CAC scores by using 2 statistically valid approaches that enabled essential adjustment for potential confounders. Moreover, the patients on hemodialysis and peritoneal dialysis in our study were relatively comparable, as this study only included patients eligible for transplantation.

In conclusion, peritoneal dialysis is not associated with less CAC nor less CAC progression than hemodialysis. This indicates that vascular calcification does not develop 
less in peritoneal dialysis. Further studies should investigate whether vascular calcification develops even more in peritoneal dialysis.

\section{Acknowledgments}

We thank the following centers for recruiting patients for the NOCTx study: Dialysecentrum Groningen, Groningen, the Netherlands; Diapriva Dialysecentrum and VU Medisch Centrum, Amsterdam, the Netherlands; Meander Medisch Centrum, Amersfoort, the Netherlands; Rijnstate Ziekenhuis, Arnhem, the Netherlands; Sint Antoniusziekenhuis, Nieuwegein, the Netherlands; Stichting Dianet Dialysecentra and Universitair Medisch Centrum Utrecht, Utrecht, the Netherlands. We thank Dr. L.J. Schurgers for providing the dp-ucMGP measurements, and Dr. I. Hoefer for the fetuin-A and osteoprotegerin measurements.

\section{Ethics Statement}

All subjects provided written informed consent. The study protocol has been approved by the Medical Ethics Committee of the University Medical Center Utrecht and the study was conducted according to the Declaration of Helsinki.

\section{Disclosure Statement}

The authors have no conflicts of interest to declare.

\section{Funding Source}

T.T.J. is supported financially by a grant from the Wellerdieck de Goede foundation with mediation of the Friends of UMC Utrecht foundation. The NOCTx study is supported by unrestricted grants from Amgen, Baxter, Fresenius Medical Care, Novartis, Roche, and Shire Pharmaceuticals. The funders had no role in study design; collection, analysis, and interpretation of data; writing the report; and the decision to submit the report for publication.

\section{Authors Contribution}

T.T.J., P.A.J., T.H., M.C.V., and B.C.V. contributed to the conception and design of this study. T.T.J., M.C.V., and B.C.V. contributed to the analysis and interpretation of the data. T.T.J. and B.C.V. drafted the manuscript. Each author contributed important intellectual content during manuscript drafting or revision and has approved of the final version of the manuscript.

\section{References}

1 United States Renal Data System: US Renal Data System 2015 Annual Data Report: Epidemiology of Kidney Disease in the United States. Am J Kidney Dis 2016;67:SA1-SA8, S1-S434.

2 Goodman WG, Goldin J, Kuizon BD, Yoon C, Gales B, Sider D, Wang Y, Chung J, Emerick A, Greaser L, Elashoff RM, Salusky IB: Coronary-artery calcification in young adults with end-stage renal disease who are undergoing dialysis. N Engl J Med 2000;342:14781483.

3 Sigrist MK, Taal MW, Bungay P, McIntyre CW: Progressive vascular calcification over 2 years is associated with arterial stiffening and increased mortality in patients with stages 4 and 5 chronic kidney disease. Clin J Am Soc Nephrol 2007;2:1241-1248.

4 London GM, Guerin AP, Marchais SJ, Metivier F, Pannier B, Adda H: Arterial media calcification in end-stage renal disease: impact on all-cause and cardiovascular mortality. Nephrol Dial Transplant 2003;18:17311740.

5 Jansz TT, Verhaar MC, London GM, van Jaarsveld BC: Is progression of coronary artery calcification influenced by modality of renal replacement therapy? A systematic review. Clin Kidney J 2018;11:353-361.

6 Vervloet MG, Sezer S, Massy ZA, Johansson L, Cozzolino M, Fouque D; ERA-EDTA Working Group on Chronic Kidney Disease-
Mineral and Bone Disorders and the European Renal Nutrition Working Group: The role of phosphate in kidney disease. Nat Rev Nephrol 2017;13:27-38.

7 Raggi P, Boulay A, Chasan-Taber S, Amin N, Dillon M, Burke SK, Chertow GM: Cardiac calcification in adult hemodialysis patients. A link between end-stage renal disease and cardiovascular disease? J Am Coll Cardiol 2002; 39:695-701.

8 Noordzij M, Korevaar JC, Bos WJ, Boeschoten EW, Dekker FW, Bossuyt PM, Krediet RT: Mineral metabolism and cardiovascular morbidity and mortality risk: peritoneal dialysis patients compared with haemodialysis patients. Nephrol Dial Transplant 2006;21: 2513-2520.

9 Korevaar JC, Feith GW, Dekker FW, van Manen JG, Boeschoten EW, Bossuyt PM, Krediet RT; NECOSAD Study Group: Effect of starting with hemodialysis compared with peritoneal dialysis in patients new on dialysis treatment: a randomized controlled trial. Kidney Int 2003;64:2222-2228.

10 Saran R, Robinson B, Abbott KC, Agodoa LYC, Bhave N, Bragg-Gresham J, Balkrishnan R, Dietrich X, Eckard A, Eggers PW, Gaipov A, Gillen D, Gipson D, Hailpern SM, Hall YN, Han Y, He K, Herman W, Heung M, Hirth RA, Hutton D, Jacobsen SJ, Jin Y, KalantarZadeh K, Kapke A, Kovesdy CP, Lavallee D, Leslie J, McCullough K, Modi Z, Molnar MZ,
Montez-Rath M, Moradi H, Morgenstern $\mathrm{H}$, Mukhopadhyay P, Nallamothu B, Nguyen DV, Norris KC, O'Hare AM, Obi Y, Park C, Pearson J, Pisoni R, Potukuchi PK, Rao P, Repeck K, Rhee CM, Schrager J, Schaubel DE, Selewski DT, Shaw SF, Shi JM, Shieu M, Sim JJ, Soohoo M, Steffick D, Streja E, Sumida K, Tamura MK, Tilea A, Tong L, Wang D, Wang M, Woodside KJ, Xin X, Yin M, You AS, Zhou H, Shahinian V: US renal data system 2017 annual data report: epidemiology of kidney disease in the United States. Am J Kidney Dis 2018;71:A7.

11 Srivaths P, Krishnamurthy R, Brunner L, Logan B, Bennett M, Ma Q, VanDeVoorde R, Goldstein SL: Cardiac calcifications are more prevalent in children receiving hemodialysis than peritoneal dialysis. Clin Nephrol 2014; 81:231-237.

12 Rroji M, Seferi S, Cafka M, Petrela E, Likaj E Barbullushi M, Thereska N, Spasovski G: Is residual renal function and better phosphate control in peritoneal dialysis an answer for the lower prevalence of valve calcification compared to hemodialysis patients? Int Urol Nephrol 2014;46:175-182.

13 Kim CD, Cho JH, Choi HJ, Jang MH, Kwon HM, Kim JC, Park SH, Lee JM, Cho DK, Kim YL: Coronary-artery calcium scores using electron beam CT in patients with chronic renal failure. J Korean Med Sci 2005;20:994999. 
14 Lee CM, Chen PW, Leung TK, Wang HJ, Kung $\mathrm{CH}$, Lin $\mathrm{YH}$, Hsiao WT, Chen YY: Comparison of coronary artery calcification in peritoneal and hemodialysis patients. J Exp Clin Med 2011;3:89-92.

15 Kidney Disease: Improving Global Outcomes (KDIGO) CKD-MBD Work Group: KDIGO clinical practice guideline for the diagnosis, evaluation, prevention, and treatment of Chronic Kidney Disease-Mineral and Bone Disorder (CKD-MBD). Kidney Int Suppl 2009;113:S1-S130.

16 Sabour S, Atsma F, Rutten A, Grobbee DE, Mali W, Prokop M, Bots ML: Multi DetectorRow Computed Tomography (MDCT) had excellent reproducibility of coronary calcium measurements. J Clin Epidemiol 2008;61: 572-579.

17 Jansz TT, Neradova A, van Ballegooijen AJ, Verhaar MC, Vervloet MG, Schurgers LJ, van Jaarsveld BC: The role of kidney transplantation and phosphate binder use in vitamin $\mathrm{K}$ status. PLoS One 2018;13:e0203157.

18 Delanaye P, Krzesinski JM, Warling X, Moonen M, Smelten N, Medart L, Pottel H, Cavalier E: Dephosphorylated-uncarboxylated Matrix Gla protein concentration is predictive of vitamin $\mathrm{K}$ status and is correlated with vascular calcification in a cohort of hemodialysis patients. BMC Nephrol 2014;15: 145.

19 Twisk J, Rijmen F: Longitudinal tobit regression: a new approach to analyze outcome variables with floor or ceiling effects. J Clin Epidemiol 2009;62:953-958.

20 Spriensma AS, Eelchout I, de Boer MR, Luime JJ, de Jong PH, Bahcecitapar MK, Heymans MW, Twisk JW: Analysing outcome variables with floor effects due to censoring: a simulation study with longitudinal trial data. Epidemiol Biostat Pu 2018;15:e12850.

21 Hokanson JE, MacKenzie T, Kinney G, SnellBergeon JK, Dabelea D, Ehrlich J, Eckel RH, Rewers M: Evaluating changes in coronary artery calcium: an analytic method that accounts for interscan variability. AJR Am J Roentgenol 2004;182:1327-1332.

22 Malluche HH, Blomquist G, Monier-Faugere MC, Cantor TL, Davenport DL: High parathyroid hormone level and osteoporosis predict progression of coronary artery calcification in patients on dialysis. J Am Soc Nephrol 2015;26:2534-2544.

23 Vervloet M, Cozzolino M: Vascular calcification in chronic kidney disease: different bricks in the wall? Kidney Int 2017;91:808817.

24 Lee H, Yoon YE, Kim YJ, Kim HL, Lee SP, Kim HK, Cho GY, Zo JH, Sohn DW: Presence and extent of coronary calcified plaque evaluated by coronary computed tomographic angiography are independent predictors of ischemic stroke in patients with suspected coronary artery disease. Int J Cardiovasc Imaging 2015;31:1469-1478.

25 Evenepoel P, Meijers BK, Bammens B, Viaene L, Claes K, Sprangers B, Naesens M, Hoekstra T, Schlieper G, Vanderschueren D, Kuypers D: Phosphorus metabolism in peritoneal dialysis- and haemodialysis-treated patients. Nephrol Dial Transplant 2016;31:1508-1514.

26 Gotch FA, Kotanko P, Thijssen S, Levin NW: The KDIGO guideline for dialysate calcium will result in an increased incidence of calcium accumulation in hemodialysis patients. Kidney Int 2010;78:343-350.
27 Simonsen O, Venturoli D, Wieslander A, Carlsson O, Rippe B: Mass transfer of calcium across the peritoneum at three different peritoneal dialysis fluid $\mathrm{Ca} 2+$ and glucose concentrations. Kidney Int 2003;64:208-215.

28 Avila M, Mora C, Prado MDC, Zavala M, Paniagua R; Mexican Collaborative Group: Osteoprotegerin is the strongest predictor for progression of arterial calcification in peritoneal dialysis patients. Am J Nephrol 2017;46: 39-46.

29 Ozkok A, Caliskan Y, Sakaci T, Erten G, Karahan G, Ozel A, Unsal A, Yildiz A: Osteoprotegerin/RANKL axis and progression of coronary artery calcification in hemodialysis patients. Clin J Am Soc Nephrol 2012;7:965973.

30 Hofbauer LC, Schoppet M: Osteoprotegerin: a link between osteoporosis and arterial calcification? Lancet 2001;358:257-259.

31 Schurgers LJ, Uitto J, Reutelingsperger CP: Vitamin K-dependent carboxylation of matrix Gla-protein: a crucial switch to control ectopic mineralization. Trends Mol Med 2013;19:217-226.

32 Price PA, Lim JE: The inhibition of calcium phosphate precipitation by fetuin is accompanied by the formation of a fetuin-mineral complex. J Biol Chem 2003;278:22144-22152.

33 Kuro-o M: Calciprotein particle (CPP): a true culprit of phosphorus woes? Nefrologia 2014; 34:1-4.

34 Miura Y, Iwazu Y, Shiizaki K, Akimoto T, Kotani K, Kurabayashi M, Kurosu H, Kuro-O M: Identification and quantification of plasma calciprotein particles with distinct physical properties in patients with chronic kidney disease. Sci Rep 2018;8:1256. 